\title{
ANALYZING LOSSES FROM HAZARD EXPOSURE: A CONSERVATIVE PROBABILISTIC ESTIMATE USING SUPPLY CHAIN RISK SIMULATION
}

\author{
Léa A. Deleris \\ Management Science and Engineering \\ Stanford University \\ Stanford, CA 94305, U.S.A.
}

\author{
Debra Elkins \\ Manufacturing Systems Research Lab \\ General Motors R\&D Center \\ Warren, MI 48090, U.S.A.
}

\author{
M. Elisabeth Paté-Cornell \\ Management Science and Engineering \\ Stanford University \\ Stanford, CA 94305, U.S.A.
}

\begin{abstract}
We present a supply chain risk analysis that is based on a Monte Carlo simulation of a Generalized Semi-Markov Process (G.S.M.P.) model. Specifically, we seek to estimate the probability distribution of supply chain losses caused by disruptions. This distribution is computed conditional on conservative hypotheses which are the following: (1) no additional risk reduction measures are implemented beyond those already in place, (2) all the products whose production has been canceled are counted as losses at their market value. The simulation thus yields conditional probabilities of loss levels that firms may reasonably use in the evaluation of business interruption costs and insurance coverage limits. The model also enables the comparison of supply chain designs based on their resilience in recovering from risk events. The approach is novel for it connects stochastic modeling of risks from an insurance perspective with supply chain network design.
\end{abstract}

\section{INTRODUCTION}

Two strategic trends, lean manufacturing and singlesourcing, have significantly changed the structure of legacy supply chains and affected their resilience to adverse events. Supply chain risk management is an emerging need for many global corporations. Many companies have achieved substantial cost reductions and enhanced operational efficiencies through the strategic redesign of their supply chain operations and the reduction of inventory levels. Yet, uncertain events, such as weather-related disruptions, labor strikes, new governmental regulations, and utility outages, can and do affect the performance of the supply chain. The evolving supply chain network structure can either exacerbate or mitigate the effects of those events on production operations and ultimately, on the performance of the firm. We focus in this paper on hazard events such as fires, floods, storms, hurricanes, thefts.

Although a specific hazard event may occur only at one location within the supply chain network, the connectivities and interdependences of plants within the network often increase the magnitude of the consequences of the hazard. For the simple supply chain represented on Figure 1 , assume that the components are single sourced to the two downstream engine plants. If the component plant suffers a business interruption, even partial, all of the downstream production (including assembly) may be affected to various degrees. Many different events may cause such a business interruption.

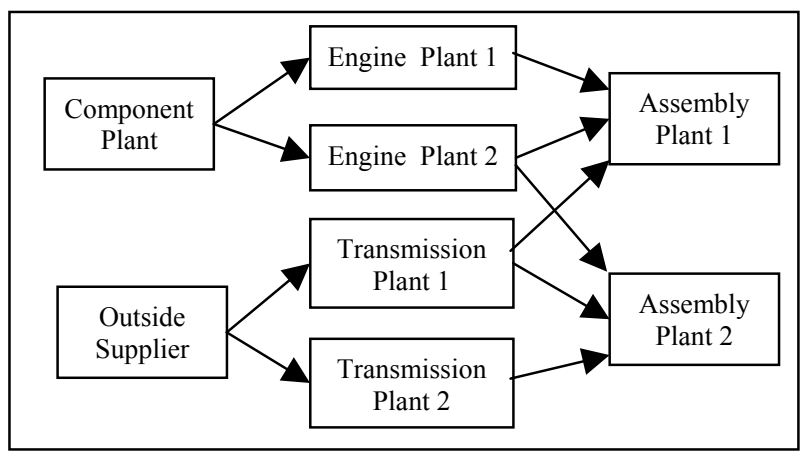

Figure 1: Schematic Representation of a Supply Chain Network

While the value of damaged buildings and broken machines is comparatively simple to assess, the estimation of the indirect consequences (loss of production) is not straightforward. Network interdependencies, characteris- 
tics of the risk, contingency plans (and their actual execution) affect the outcome. Some disasters are reduced to mere incidents (lucky near-misses), while other operational problems can be more damaging.

We therefore recognize the need to further study the risk of business interruption in supply chain networks. In order to support decision making, we believe that a quantification of the risk is necessary. Beyond qualitative risk assessment, risk quantification permits setting priorities under financial constraints. As an initial step toward supply chain risk quantification, we propose a modeling approach that estimates the risk exposure of a supply chain structure from a specific hazard. This risk exposure is computed conditional on conservative hypotheses which are the following: (1) no risk reduction measures are implemented beyond those already in place, (2) all the products whose production has been canceled are counted as losses at their market value. In reality, some mitigation measures, such as finding an alternative source of supply, can attenuate considerably the losses. Also, it may be that inventories are such that delayed production does not result in a total loss of sales. The model that we present is relevant for the estimation of a conservative value of the losses that the firm may face. Contingency plans and reaction schemes can then be evaluated by comparison to the results of this conservative, no contingency, base case estimation.

\section{BACKGROUND: MOTIVATION AND RELATED RESEARCH}

Our research is motivated by a renewed corporate interest in managing supply chain risks. Companies are more aware of and sensitive to global supply chain interruptions than in the past, particularly following the September 11, 2001 terrorist attacks on the United States, and the October 2002 West Coast longshoreman's labor strike. These major disruptions have drawn attention to the vulnerabilities of supply chains, predominantly for industries that are operating lean supply chains. In those industries, the benefits of a single-string supply chain design may be in part mitigated by the risk of interruption in the operations of that single source.

In addition, supply chain management literature has begun to focus on supply chain vulnerabilities. Deleris (2003) and Elkins (2003) have explored the application of risk and decision analysis methods to manufacturing and supply chain operations. Callarman (2003) describes a high-level qualitative risk model that examines supply chain risk by considering different flow disruptions (material, information, knowledge, cash, and capital equipment). Chapman et al. (2002), Helferich and Cook (2002), and Peck and Juttner (2002) discuss how to identify and manage supply chain vulnerabilities. The NACFAM report (2003) and the Cranfield University report (2002) both comment on the heavy reliance of corporate supply chains on public transportation, utilities, and communications infrastructures. Sheffi (2001) examines how companies can organize supply chain operations to meet challenges of operating under increased security regimes and terrorist threats. Mansfield (2003) presents the military perspective on supply chain risk management, and highlights the need for flexibility through dynamic reconfigurable supply chains. Lindroth and Norman (2001) discuss risk sharing among supply chain partners, and present a case study on risk sharing from the telecommunications industry. Holmes (2003) comments on the increasing interaction between insurance managers and supply chain operations managers and suggests that risk management decisions be jointly reviewed by supply chain and riskinsurance experts. Finally, Lester (2002) points out that single sourcing can work if properly managed, and comments that "you can put all your eggs in one basket, as long as you watch the basket very carefully".

The remainder of the paper is organized as follows. Section 3 describes our model in general terms. Section 4 details the application of this model to a specific hazard, describing the inputs, outputs and presenting illustrative results. A discussion is found in Section 5. We conclude in Section 6.

\section{GENERAL RISK SIMULATION APPROACH}

\subsection{Introduction to the Model}

The goal of our model is to assess the risk exposure of a given supply chain due to a specific hazard, for example fires in production plants. The general idea behind our model is that any hazard event creates local shock (disruption i.e., the plant may not be fully operational after the event) on at least one of the locations of the supply chain. If there is no slack or substitute, this local event generates further disruptions to downstream locations and reduces the supply chain output. We compute the effect of the hazard over a time period by estimating both direct and indirect losses. Direct losses are the losses incurred due to the immediate effects of an event (e.g. property damage costs) and indirect losses are the losses caused by the reduction in supply chain output. Direct and indirect losses are aggregated into a total loss measure for the considered time period. The loss measure may be based on cost, lead time, or customer service rate. In this paper, we chose for illustration the study of fire hazard within a supply network and we focus on the costs as an aggregate measure of all direct and indirect losses (See Section 4).

\subsection{Description of the Model}

The overall model has four parts. Each of them is described in greater details further.

- Hazard Model: Set of plant-specific models of the sequence of hazard events occurrences and severity described as dependent stochastic processes. 
- Operations Model: Model of the manufacturing operations / supply chain network.

- G.S.M.P. Model: Integration of both hazard and operations models into a General Semi Markov Process (G.S.M.P.) model (A continuous stochastic model representing system transitions among states over time).

- Monte Carlo Simulation: Simulation of the G.S.M.P. model sample path to obtain the probability distribution of the total losses per time unit (here: ninety days).

The hazard model describes, for each plant in the supply network, the occurrences of the hazard events as a stochastic process, and their severity levels (for instance cost and downtime) conditional on an event. Geographic dependencies among plants (for weather related hazard for instance), as well as plant characteristics (e.g., building material or activity) have to be accounted for. Many sources of information such as expert opinion and statistical time series are generally available to assess the probability distributions for the hazard of interest. In short, this model describes at the plant level the sequence of disruptions (and their consequences) that the network endures during one time period.

The operations model describes in details the supply chain structure and in particular, interactions among locations. This model is used to quantify how perturbations of the network caused by the hazard sequence affect the other plants within the network and reduce the supply chain output. Therefore the operations model captures the compounded effects (direct and indirect) of hazard disruptions. This model can be developed separately from the hazard event occurrence model, given the knowledge of the plants that constitute the supply chain network. The choice of the type of model depends on the supply chain output loss measure. For a measure based on cost, a product-mix model (describing the inputs and output of the chain) may be sufficient.

The General Semi Markov Process (G.S.M.P.) integrates both hazard and operations models to describe the evolution of the network as a stochastic process. Recall that a G.S.M.P. model is a continuous time model that permits sample path simulation of a stochastic system that represents the transitions from state to state triggered by the events. In this application of the G.S.M.P. framework, the system analyzed is the supply network, triggering events are either hazard occurrence or recovery from them, and the state of the network is described by a vector representing state of operations at each plant location within the network. The interested reader can find more information on G.S.M.P. in Shedler (1993). Specifically, in our model, the G.S.M.P. uses the hazard model to generate input for modeling of the times between hazard occurrences and for the severity of each occurrence, which can be time and lo- cation dependent (Note that, for simplicity in what follows, we assume that the frequencies and severities of hazard events are the same for all locations and that the severity is time independent.). The operations model is used to determine the effect of an event on each location of the network and to evaluate the supply chain losses. The simulation of the G.S.M.P. provides a sample path of the states of the supply chain during one time period, and enables us to assess the total losses.

The Monte Carlo simulation of the G.S.M.P. iterates this assessment many times in order to obtain enough data points to derive the probability distribution of the total losses for the time period considered. From this distribution, we derive the corresponding complementary cumulative distribution function, often referred to as a "risk curve" in risk analysis (The complementary cumulative distribution function $\mathrm{G}(\mathrm{x})$ is defined as $1-\mathrm{F}(\mathrm{x})$ where $\mathrm{F}(\mathrm{x})$ is the cumulative distribution function). The risk curve thus represents the probability of exceeding a given level of losses per time period.

The general approach is summarized in Figure 2.

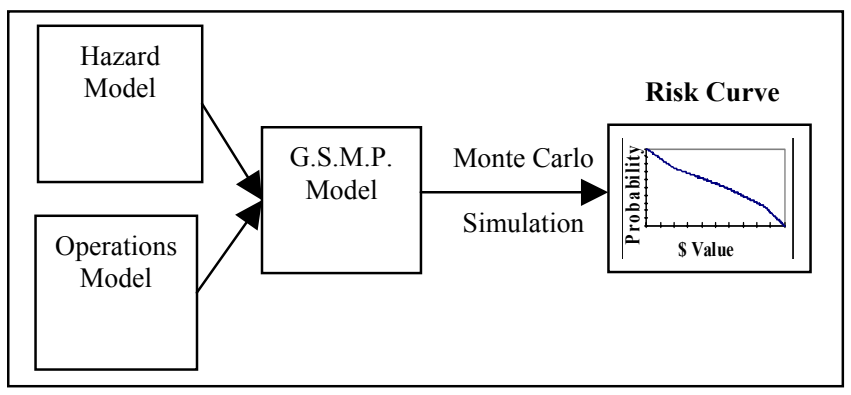

Figure 2: General Risk Simulation Modeling Approach

\section{APPLICATION TO FIRE RISK IN A MANUFACTURING NETWORK}

To demonstrate the feasibility of the method, we study the effect of fire hazard on a large U.S. manufacturing supply network. This section describes the details of our application. For confidentiality reasons, the numerical results presented are derived from a simplification of the network on which the actual implementation is performed.

\subsection{Implementation Settings}

The large supply chain network that we analyze is composed of more than 80 plants (5 different categories of plants e.g., components, subassembly A, subassembly B, subassembly $\mathrm{C}$, assembly). The time period chosen for the analysis is a 90 -day quarter.

The main metric to estimate supply chain losses is total financial loss per operating quarter, which is defined as the sum of lost production costs and property damage costs caused by fire hazards. As secondary loss metrics, we 
evaluate lost production per quarter (i.e., number of items not produced per quarter because of the fire events) and mean downtime per plant type. The lost production per quarter (not reported in this paper) is used as a surrogate measure for financial loss and serves to validate the main outputs for consistency. The mean downtime per plant is useful to understand and quantify the indirect effects of the network interdependencies. Managers can use that measure to prioritize investments in risk reduction efforts.

In our implementation, we assume that the same type of fire (both in occurrence and severity) occurs independently at each plant within the network. Hence, we do not differentiate the plants by type. Fire hazards are assumed to occur according to a Poisson process. The severity (damage cost and downtime) of each fire is assessed from a discrete distribution (e.g., Small, Medium, and Large). Both the parameter of the Poisson process and the severity distribution are based on data analysis and expert opinion.

We use a product-mix model as our operations model, i.e. to translate the loss of production volume due to a local business interruption into monetary loss downstream. We do not include any transportation activity in our operations model, thus implicitly assuming that transportation is always operational.

The notation for the G.S.M.P. model and the simulation algorithm are provided in the appendices. Next we describe output of the simulation.

\subsection{G.S.M.P. System Simulation}

Each sample path of the G.S.M.P. is completely described by the sequence of states $\left\{\vec{S}^{n}\right\}_{n=0}^{\infty}$ and the sequence of state transition times $\left\{T_{n}\right\}_{n=0}^{\infty}$. Note that any time where all plant locations return to normal operating conditions is a point of regeneration, where the probabilities that describe the evolution of the system in the future are independent of the system behavior in the past. We start each simulation iteration at $T_{0}=0$ with $\vec{S}^{0}=(1,1 \ldots 1)$ with probability 1 , so that the G.S.M.P. begins at a point of regeneration. Thus, in our implementation, the G.S.M.P. is a nondelayed regenerative stochastic point process. While we do not use this regenerative property, it may prove useful for future uses of this model, for instance to study times between returning to normal operations (i.e. when all locations are working).

\subsection{Simulation Results}

Again, while we implement the method on an actual supply chain, all numerical results presented here are for illustrative purposes only, and demonstrate how one might use the simulation to analyze and communicate risk exposure. The results of the simulation are hypothetical, but represent the kind of results that may be obtained from actual data.
The number of iterations of the Monte Carlo simulation is arbitrarily chosen to be 1,000 . We perform a visual verification of the convergence of our measures. Future work may address risk simulation convergence issues more thoroughly. This paper focuses on the use the G.S.M.P. algorithm for risk assessment and on its value when applied to real-world problems.

In our application, the total quarterly losses is our primary measure for risk. The results, which are shown on Figure 3, provide a distribution of the losses due to fire hazards, from $\$ 0$ to more than $\$ 1 \mathrm{M}$ per 90 -day quarter. The median of total losses is approximately $\$ 100 \mathrm{~K}$ per quarter, meaning that there is $50 \%$ chance that the total quarterly losses due to fire hazard will exceed $\$ 100 \mathrm{~K}$. One insight from these results, which holds for actual data, is that property damage costs are negligible compared to lost production cost. While this relationship is to be expected, the simulation provides a numerical estimation of the relative importance of the two types of losses. The traditional focus of insurance has been to protect the property value of assets from catastrophic risks, the simulation shows that a company can also benefit from actively reducing its exposure to sale losses through mitigation actions at the supply chain level.

\section{Loss Exceedance Probability (Risk Curve) for Total Cost}

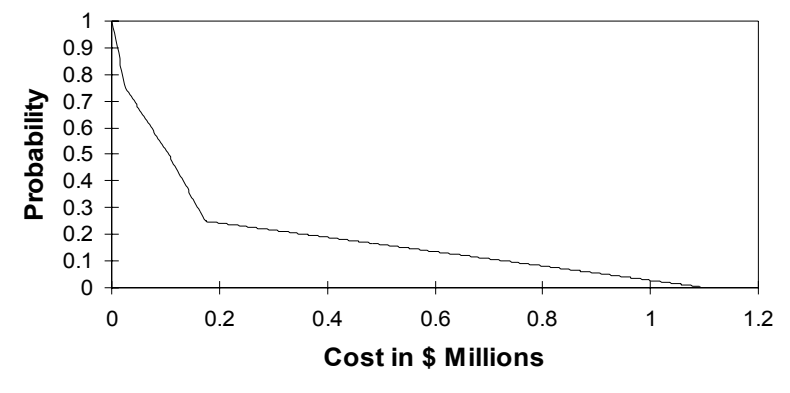

Figure 3: Loss Exceedance Probability (Risk Curve) for Total Cost of Risk Events per 90-Day Quarter

We also report the mean downtime per plant category on Figure 4 (the number of plants in each category is noted in parentheses). This metric captures the effect of supply chain network interdependences and is obtained by averaging mean down time per plant over each plant category. Mean downtime for a specific plant is computed as the average of the percentage of plant downtime during a simulated 90-day quarter. The computed levels for downtime can be thought of as a reference vulnerability metric, against which alternative supply chain designs may be compared. We observe that assembly plants, which are the most downstream within the network, suffer higher downtime than other category of plants. We also note that the outside supplier has more downtime compared to internal 
engine, transmission, or component plants, possibly signaling a supplier reliability problem.

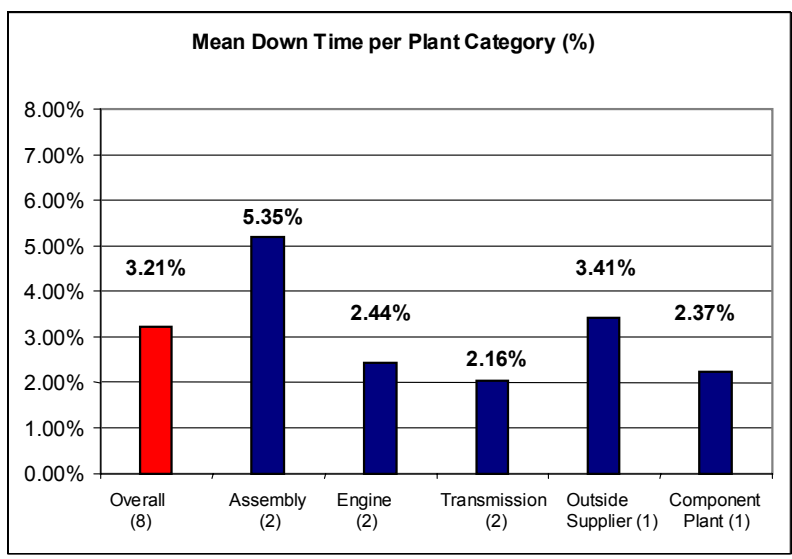

Figure 4: Effect of Fire Hazard on Plant Category Downtimes

\section{DISCUSSION}

\subsection{General Comments about the Model}

In this research, we choose to use a G.S.M.P. to model the evolution of the supply chain network under a stochastic risk load. While the G.S.M.P. is more general than a Markov Chain stochastic model, it is also more complex to describe and to implement, and requires longer computing run-time. In addition, as opposed to Markov chain, it does not necessarily yield closed form limiting solutions. One advantage of the G.S.M.P. framework, however, is that it incorporates naturally any kind of hazard events because it allows for multiple triggering events and for general distribution for the times between transitions. This flexibility enables, for example, to have non-Poisson hazard occurrences and deterministic recovery times.

We note that G.S.M.P.s have been extensively used in modeling stochastic risk processes, and that numerous simulation models of supply chain networks exist. Our approach is innovative because it connects risk modeling based on insurance data with the structure of the supply chain networks.

Another important difference of our approach from the mainstream of the supply-chain literature is that we do not seek to optimize the supply chain, but to obtain, as a base case, a probabilistic description of its performance for a specific risk in order to support management decision for risk reduction or supply chain design.

Finally, while this research is aimed at supply chain risk management, the approach that we present focuses principally on risk assessment. To reduce the risk exposure, changes in insurance programs and supply chain network design can be considered, for instance, reinforcing the loss prevention equipment at a specific set of plants, or reevaluating the terms of the insurance policy with regards to the hazard considered.

\subsection{Limitations}

While our method successfully connects insurance risk modeling with supply chain network structures, we acknowledge that the model has some limitations. First, we do not take into account any mitigation or loss control efforts to respond to risk events. In addition, we do not model inventory as a buffer for production interruption. Finally, we assume that all units that are not built are lost sales. Thus, under those limitations, the results from the implementation of the approach provide a conservative estimate of risk exposure. This fact does not lessen the value of our analysis. It can be easily introduced in the model if needed. The cost estimation is useful for planning insurance coverage relative to different types of risks.

Furthermore, the approach that we present in this paper relies explicitly on a descriptive model of the supply chain (the operations model). While the simple productmix model is sufficient in our implementation, it deliberately ignores the transportation links. We do not suggest any standard supply chain model for risk assessment purposes but note that a standard model supply chain description is valuable. Such a model should allow to:

- Include many tiers of the supply chain, and many suppliers/customers within each tier. This would extend the analysis backward or forward to explore risks affecting upstream suppliers, or downstream distribution and warehousing operations.

- Add intermediate degrees in production levels for the plants within the production network. In our implementation, plants are either fully operational or not operating at all. It would be more realistic to describe the operating state of each plant for instance through the percentage of its production capacity available.

- Incorporate transportation modes and logistics routes so that logistics risks can be further explored. Including transportation links explicitly in supply chain model is critical.

\subsection{Further Use of the Model}

The model architecture permits the independent and parallel development of the hazard model and operations model. This flexibility is one of the strength of the model.

First, the current simulation model can be applied to a variety of hazards, if the probability of risk events and the associated loss severity can be described using probability distributions. Therefore, it is possible to study similarly the impact of utility outages (gas, water, electricity), labor strikes or sequences of natural disasters or catastrophic 
events. This simply entails modifying the hazard model accordingly. We believe that assessing risk exposure from various hazards using the same operations model constitutes a reliable basis for comparison among hazards.

Second, while holding the hazard model unchanged, supply chain analysts can modify the operations model to investigate the risk exposure of different network topologies (i.e., supply chain designs).

Furthermore, it is possible to extend the hazard model to include merging of distinct risk processes, so that the overall (i.e., to many different hazards) risk exposure of the supply chain can be estimated. The approach is scalable in the sense that it is possible to include many hazards simultaneously instead of one at a time. For that matter, only the hazard model has to be modified. However, stochastic modeling of various risks is challenging, especially if one wants to capture the dependencies among risks.

To conclude, the proposed approach is useful:

- To compare the risk exposure from different hazards and support resources allocation decisions.

- To evaluate the efficiency of alternative supply chain structures for risk mitigation.

- To assess the overall exposure of a supply chain structure from a set of different hazards.

\section{CONCLUSION}

We have described an approach based on a Generalized Semi-Markov Process (G.S.M.P.) including Monte Carlo simulation to the assessment of the disruption caused by a specific type of hazard on a supply chain. The model provides decision support for managers to evaluate a firm's risk exposure. The model estimates the probability distribution of the loss in supply chain output caused by the occurrence of hazards within the supply network. The model can be further used to assess the performance of alternative supply chain structures.

\section{APPENDIX A: G.S.M.P. NOTATION}

$N=$ number of plant locations in the operations model.

$S=$ set of system states.

$\vec{s}^{n}=$ current state of the system after the $n^{\text {th }}$ state transition. $\vec{s}^{n} \in\{0,1\}^{N}$ where $s_{i}^{n}=0$ if production at plant $i$ has been directly interrupted because of a risk event, and $s_{i}^{n}=1$ if plant $i$ is operating normally. $\vec{s}^{n}$ does not incorporate supply chain interdependencies (i.e., indirect disruptions). Here we use a simple binary description of the state of the system.

$E$ = finite set of all possible events that triggers a transition from one system state to another.

$e_{\text {risk }}=$ event representing hazard occurrence in one of the plants of the production network. $e_{i}=$ event of plant $i$ recovering normal production operations.

$E\left(\vec{s}^{n}\right)=$ set of all events eligible to occur after the system makes the $n^{\text {th }}$ state transition. By definition, $E\left(\vec{s}^{n}\right) \subseteq E$. Note that plant $i$ can recover normal operations (event $e_{i} \in E\left(\vec{s}^{n}\right)$ ) if and only if $s_{i}^{n}=0$, (i.e., production at plant $i$ has been interrupted by a risk event).

$\vec{c}^{n}=n^{\text {th }}$ clock reading vector. $\vec{c}^{n}=\left(c_{r i s k}{ }^{n}, c_{1}{ }^{n} \ldots\right.$ $c_{N}{ }^{n}$ ). Each coordinate represents the time until event $e_{i}$ occurs after the transition of the system into state $\vec{s}^{n}$.

$P\left\{\vec{s}^{(n+1)} \mid \vec{s}^{n}\right.$, event $\left.e\right\}=$ probability of transition to system state $\vec{s}^{(n+1)}$ given that the current system state is $\vec{s}^{n}$ and event $e \in E\left(\vec{s}^{n}\right)$ occurs. If the event that occurs is $e_{i}$ (i.e., plant recovering from production interruption), then $P\left\{\vec{s}^{(n+1)} \mid \vec{s}^{n}\right.$, event $\left.e_{i}\right\}=1$, where $\vec{S}^{(n+1)}=\left(s_{1}^{n}, s_{2}^{n}, \ldots, s_{i-1}^{n}, 1, s_{i+1}^{n}, \ldots, s_{N}^{n}\right)$.

$r\left(\vec{s}^{n}, e\right)=$ event recovery rate (i.e., clock speed), dependent on the current state $\vec{s}^{n}$ and the system state transition triggering event $e$. For this simulation model, we set $r\left(\vec{s}^{n}, e\right)=1$ for all states $\vec{s}^{n}$ and all events $e$. It may be useful to set clock rates $r\left(\vec{s}^{n}, e\right)>1$ to model situations where additional resources are allocated to mitigate a risk event $e$ depending on the current state $\vec{s}^{n}$. If the rate of recovery is increased, additional mitigation costs should be accounted for.

$T_{n}=$ time of the $n^{\text {th }}$ state transition, with $T_{0} \leq T_{1} \leq T_{2} \leq \ldots$. Time $T_{0}=0$ represents the beginning of the simulated 90 -day period.

$T_{n}-T_{n-1}=$ time between the $(n-1)^{\text {st }}$ and $n^{\text {th }}$ state transitions. Also defines the sojourn or holding time in state $\vec{s}^{n-1}$ before transitioning to state $n$.

\section{APPENDIX B: G.S.M.P. ALGORITHM}

1. System initialization

a. Set $\vec{s}^{0}=(1,1, \ldots, 1)$ so that each plant starts each iteration in normal operating conditions.

b. Set $T_{0}=0$.

c. For each event $e_{i} \in E\left(\vec{s}^{0}\right)$, generate a clock reading $c_{i}^{0}$ as a randomly distributed time to sojourn in state $\vec{s}^{0}$ until event $e_{i}$ should cause the process to transition out of state $\vec{s}^{0}$. Set $c_{i}^{0}=0$ for those events $e_{i} \notin E\left(\vec{s}^{0}\right)$. Note that at that point the only element in $E\left(\vec{s}^{0}\right)$ is $e_{r-}$ isk. When a risk event occurs somewhere in the production network, then assign the event to that plant location $i$, where the event occurred. Note that the assignment of risk event to plant location depends on the type of risk event. For some risks, such as plant fires, each plant may be equally likely to experience the risk, and 
thus assigning a risk event to a plant location can be done using a discrete uniform $(1, N)$ distribution. For other risks, such as flood or earthquake, some plant locations may have higher probabilities of experiencing the risk event. In this case, the probability distribution for assigning the risk event to a particular plant may be specified using a discrete but nonuniform distribution. The associated risk event severity is assigned according to a loss severity distribution, which may be dependent on plant location $i$, and time of event occurrence.

d. Set the rate of recovery $r\left(\vec{s}^{n}, e\right)=1$ for all $\mathrm{n}$ and all possible events $e$.

Repeat Steps 2-7 for $\mathrm{n}=1,2,3, \ldots$

2. Identify when and why the $n^{\text {th }}$ state transition will occur.

a. Determine $T_{n}$, the time of the $n^{\text {th }}$ state transition out of state $\vec{s}^{n-1}$.

$$
T_{n}-T_{n-1}=\min _{\left\{\text {indices } i \mid e_{i} \in E\left(\vec{s}^{n-1}\right)\right\}}\left\{\frac{c_{i}^{n-1}}{r\left(\vec{s}^{n-1}, e_{i}\right)}\right\}
$$

b. Determine the subset of events that will actually trigger the $n^{\text {th }}$ state transition.

$$
E^{*}\left(\vec{s}^{n-1}\right)=\left\{\text { events } e_{i} \mid \frac{c_{i}^{n-1}}{r\left(\vec{s}^{n-1}, e_{i}\right)}=T_{n}\right\}
$$

3. Identify the next system state $\vec{s}^{n}$ into which the system will transition.

a. If the state transition triggering event is $e_{\text {risk }}$, i.e., a risk event occurs somewhere in the production network of plants, then assign the risk event to a plant location $i$, where the event occurred. Update $s_{j}^{n}=s_{j}^{n-1}$ for $j \neq i$, and $s_{i}^{n}=$ 0 . The risk event severity is assigned according to the conditional severity distribution, which may be dependent on plant location $i$, and time of event occurrence $T_{n}$.

b. If the state transition triggering event is $e_{i}$, plant $i$ recovers from a risk event, and returns to normal operating conditions. $s_{j}^{n}=s_{j}^{n-1}$ for $j \neq i$, and $s_{i}^{n}=1$.

4. Update the set of new events that could trigger the $(n+1)^{s t}$ state transition.

a. Define new event set

$$
N\left(\vec{s}^{n}\right)=E\left(\vec{s}^{n}\right)-\left(E\left(\vec{s}^{n-1}\right)-E^{*}\right) .
$$

b. For each event $e_{i} \in N\left(\vec{s}^{n}\right)$, generate a clock reading $c_{i}^{n}$ as the randomly distributed time to sojourn in state $\vec{s}^{n}$ until event $e_{i}$ should cause the process to transition out of state $\vec{s}^{n}$.

5. Update the set of old events that could trigger the $(n+1)^{s t}$ state transition.

a. Define old event set

$$
O\left(\vec{s}^{n}\right)=E\left(\vec{s}^{n}\right) \cap\left(E\left(\vec{s}^{n-1}\right)-E^{*}\right) .
$$

b. Update times of old events with new clock readings. Set

$$
c_{i}^{n}=c_{i}^{n-1}-\left(T_{n}-T_{n-1}\right) \cdot r\left(\vec{s}^{n-1}, e_{i}\right) .
$$

6. Cancel event(s) $e_{i}$ that caused the state transition from state $\vec{s}^{n-1}$. Set $c_{i}^{n}=0$ for these event indices.

7. Move to state $\vec{s}^{n}$ and time $T_{n}$.

a. If $T_{n} \leq$ run length of 90 days, set $n=n+1$ and return to step 2 .

b. If $T_{n}>$ run length of 90 days, STOP algorithm.

\section{REFERENCES}

Callarman, T. 2003. Extended Enterprise Risk Management \& Continuity. The Enterprise and Supply Chain Risk Management. \& Continuity Forum. National Center for Manufacturing Sciences. Ann Arbor, Michigan.

Chapman, P., Christopher, M., Juttner, U., Peck, H., Wilding, R. 2002. Identifying and Managing Supply chain Vulnerability. Cranfield Centre for Logistics and Transportation. Cranfield School of Management. United Kingdom.

Cranfield University. School of Management. 2002. Supply Chain Vulnerability. Executive Report prepared for Department for Transport, Local Government and the Regions, Home Office, Department of Trade and Industry. United Kingdom.

Deleris, L. 2003. A Risk Analysis Perspective on Supply Chain Management of Manufacturing Companies. Second Year Paper, Department of Management Science and Engineering, Stanford University, Stanford, California.

Elkins, D. 2003. A Framework for Business Interruption Risk Analysis. Research Report MSR-051, General Motors R\&D Center, Warren, Michigan.

Helferich, O. K., Cook, R. L. 2002. Securing the Supply Chain. White Paper: Management Report. Council of Logistics Management. Oakbrook, Illinois.

Holmes, C. 2003. Supply Chain Risk Management: A Significant Convergence of Operations and Risk Manage- 
ment Concerns. The Enterprise and Supply Chain Risk Management. \& Continuity Forum. National Center for Manufacturing Sciences. Ann Arbor, Michigan.

Lester, T. 2002. Making it Safe to Rely on a Single Partner. Financial Times.

Lindroth, R., Norman, A. 2001. Supply Chain Risks and Risk Sharing Instruments - An Illustration from the Telecommunications Industry. In Logistics Research Network $6^{\text {th }}$ Annual Conference Proceedings. 297-306.

Mansfield, Jr., Brig. Gen. R. E. 2003. Thinking About Risk in the Military Supply Chain. The Enterprise and Supply Chain Risk Mgmt. \& Continuity Forum. National Center for Manufacturing Sciences. Ann Arbor, Michigan.

National Coalition for Advanced Manufacturing (NACFAM). 2003. Critical Infrastructures and U.S. Manufacturing: Assessing the Vulnerabilities to Disruptions from Terrorism. NACFAM Washington, D.C.

Peck, H., Juttner, U. 2002. Risk Management in the Supply chain. Focus. Cranfield Centre for Logistics and Transportation. Cranfield School of Management. United Kingdom.

Shedler, G. 1993. Regenerative Stochastic Simulation. San Diego: Academic Press.

Sheffi, Y. 2001. Supply Chain Management under the Threat of International Terrorism. International Journal of Logistics Management. 12 (2) 1-11.

\section{AUTHOR BIOGRAPHIES}

LÉA A. DELERIS is a Ph.D. candidate in Management Science and Engineering at Stanford University. She holds an M.S. in Management Science and Engineering from Stanford University, a Diplôme d'Ingénieur from Ecole Polytechnique, and a Diplôme d'Ingénieur from Ecole Nationale des Ponts et Chaussées. Her research interests include risk analysis and the decision sciences. She has worked for several years with General Motors R\&D on modeling and analysis for supply chain risk and business interruption management. Her dissertation is focusing on early warning systems for strategic decisions. Her e-mail address is <ldeleris@stanford.edu>.

DEBRA ELKINS Ph.D. is the Research Program Manager and technical lead for Enterprise Risk Management research at General Motors R\&D Center in Warren, Michigan. Her research interests include manufacturing and supply chain risk modeling and analysis, decisionmaking under uncertainty, computational issues in stochastic processes and applied probability, and enterprise scale simulation. She has served as an industry technical expert for the Dept. of Homeland Security University Based Centers of Excellence, and recently briefed the U.S. National Defense University / Industrial College of the Armed Forces on global manufacturing and supply chain risks. Elkins holds a B.S. in Mathematical Physics from Sweet
Briar College, Virginia, M.S. in Mathematics - Operations Research, and Ph.D. in Industrial Engineering - Operations Research from Texas A\&M University - College Station. She also enjoys teaching Production Operations, and serves as an adjunct lecturer in the School of Management at the University of Michigan - Dearborn. Her e-mail address is <debra.elkins@gm.com>.

M. ELISABETH PATÉ-CORNELL Ph.D. is the Burt and Deedee McMurtry Professor in the School of Engineering and has been chair of the Department of Management Science and Engineering at Stanford University since its creation in January 2000. Her primary areas of teaching and research are engineering risk analysis and risk management, decision analysis, and engineering economy. Her research, in recent years, has focused on the extension of probabilistic risk analysis models to include organizational factors with application to a wide variety of problems such as the management of the tiles of the space shuttle, offshore platforms during oil and gas production, and anesthesia during surgery. She is currently working on mathematical models that allow management of programmatic risks for the development of safety-critical systems, for instance in the space industry. She is a member of the President's Foreign Intelligence Advisory Board, of the National Academy of Engineering and of its Council, and of the California Council on Science and Technology, and formerly a member of the Air Force Science Advisory Board and of the NASA Advisory Council. She is a past president and a fellow of the Society for Risk Analysis. She is currently chair of the Committee on Research of Stanford University. Paté-Cornell received her Engineer Degree in Computer Science in 1971 from the Institut Polytechnique of Grenoble, France, a master's degree in operations research in 1972 and a $\mathrm{PhD}$ in Engineering-Economic Systems in 1978, both from Stanford University. 